\title{
THE DESIGN OF A COMPACT RFQ NEUTRON GENERATOR
}

\author{
R. W. HAMM \\ R\&M Technical Enterprises, Inc. 4725 Arlene Place, Pleasanton, CA 94566, USA \\ rmtech@comcast.net \\ R. BECKER \\ IAP, Goethe-Universität, Max von Laue-Straße 1, 60438 Frankfurt am Main, Germany \\ rbecker@physik.uni-frankfurt.de \\ Published 25 February 2014
}

\begin{abstract}
The output and target lifetime of a conventional electrostatic neutron generator are limited by the voltage stand-off capability and the acceleration of molecular species from the ion source. As an alternative, we suggest that the deuterium beam achievable from a compact high intensity ECR source can be injected directly into a compact RFQ to produce a more efficient compact neutron production system. Only the $\mathrm{d}^{+}$ions are accelerated by the RFQ, which can also produce much higher output energies than electrostatic systems, resulting in a higher neutron output with a longer target lifetime. The direct injection of the beam makes the system more compact than the multielement, electrostatic systems typically used for extraction of the beam and subsequent transport and matching into the RFQ. We have designed and optimized a combined extraction/matching system for a compact high current deuterium ECR ion source injected into a high frequency RFQ structure, allowing a beam of about $12 \mathrm{~mA}$ of $\mathrm{d}^{+}$ions to be injected at a modest ion source voltage of $25 \mathrm{kV}$. The end wall of the RFQ resonator serves as the ground electrode for the ion source, resembling DPI (direct plasma injection). For this design, we used the features of the code IGUN to take into account the electrostatic field between the ion source and the RFQ end wall, the stray magnetic field of the ECR source, the defocusing space charge of the low energy deuteron beam, and the rf focusing in the fringe field between the RFQ vanes and the RFQ flange.
\end{abstract}

Keywords: Neutron source; ECR source; RFQ.

PACS numbers: 29.20.Ej, 29.25.Dz, 29.25.Nj

\section{Introduction}

The radiofrequency quadrupole (RFQ) accelerator has a number of advantages as a neutron generator over other accelerator-based devices such as sealed tubes, electrostatic accelerators, and cyclotrons. These compact rf linacs can be used to efficiently produce beams of protons and deuterons up to a few MeV. As shown in Fig. 1, these low energy beams can be used with a number of target materials to produce high neutron output

This is an Open Access article published by World Scientific Publishing Company. It is distributed under the terms of the Creative Commons Attribution 3.0 (CC-BY) License. Further distribution of this work is permitted, provided the original work is properly cited. 
fluxes. ${ }^{1}$ As a result of the many reactions available, these systems can produce a wide range of output neutron spectra. ${ }^{2}$ The high current capability and rugged construction make them well-suited for many industrial applications requiring neutrons. A number of commercial systems are currently available from AccSys Technology, Inc., ${ }^{3}$ but these units are not yet small enough to replace conventional sealed tube neutron generators.

However, several new technical innovations now make it possible to produce a very compact unit that can indeed replace existing conventional $(\mathrm{d}, \mathrm{d})$ neutron generator systems. The configuration presented in this paper uses a compact permanent magnet ECR ion source mounted directly onto the input flange of a very short RFQ linac. A revolutionary new extraction system is used to inject the deuterons directly from the ion source into the RFQ structure without the standard matching lens used in conventional RFQ linacs. This small $425 \mathrm{MHz}$ RFQ produces a $500 \mathrm{keV}$ deuteron beam in less than $0.5 \mathrm{~m}$ and can be powered by a very compact solid-state rf power system. The output from this RFQ is pulsed with a constant peak beam current, but the pulse width and pulse repetition rate can be varied over a wide range to provide a varying intensity of neutron flux. As indicated in Fig. 1 by the circled region, the $500 \mathrm{keV} \mathrm{d}{ }^{+}$beam can be used with a variety of targets $\left(\mathrm{Be}, \mathrm{D}_{2}\right.$ or $\left.\mathrm{Li}\right)$ to produce neutron yields in the range of 1.5-2.5 x $10^{7}$

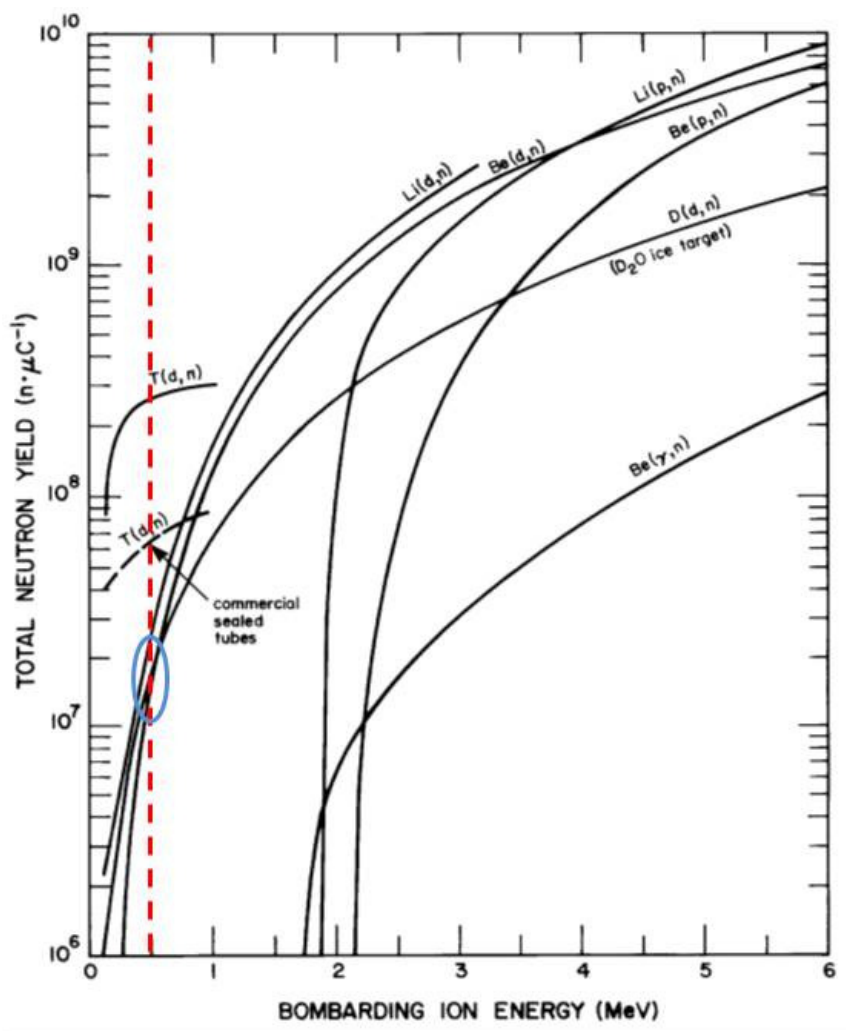

Fig. 1. Neutron yields for low energy particle beam reactions. ${ }^{1}$ 
neutrons $/ \mu \mathrm{A}$. The high current ion beam exits the RFQ linac with a low divergence in both planes, which makes it possible to transport the beam in a short drift tube from the accelerator to a remote target, resulting in smaller shielding and neutron moderator requirements. This makes it ideal for generating thermal neutrons.

The major innovations that make this compact RFQ-based neutron generator possible are the $\mathrm{d}^{+}$ion injector that uses a mini-ECR ion source and a new combined extraction/injection system with the end wall of the RFQ serving as the extraction electrode, and the very short, efficient RFQ that accelerates a $12 \mathrm{~mA}$ pulsed beam and only uses a small solid-state rf power system. Commercial systems for cooling and vacuum can be used and the system can be controlled with LabVIEW ${ }^{\circledR}$ software on a laptop computer. All of the electronics and rf components can be mounted in the support frame of the RFQ to make it a single small transportable unit. The new technology used for this system can be extended to higher energy RFQs to produce larger systems capable of neutron outputs of two orders of magnitude more, but these are not compact systems packaged into a single unit.

\section{Ion Source}

The data from the duoplasmatron ion source available from AccSys Technology was originally used for the beam extraction and matching calculations, as it delivers $15 \mathrm{~mA}$ of deuterons at $25 \mathrm{kV}$. A conservative $12 \mathrm{~mA}$ of deuterons was assumed for this design. This duoplasmatron is a mature ion source, but it can easily be replaced by a compact $2.45 \mathrm{GHz}$ ECR source, development of which is being pursued at several places around the world. Hence the ion injector proposed for this system uses such a mini-ECR source operating with permanent magnets to produce the magnetic fields in the source ionization region. The microwave power to the mini-ECR source uses a high voltage break in a waveguide to place the microwave system at ground potential, avoiding all the power supplies that must be at high voltage to operate the duoplasmatron. A tuner can be used to match the waveguide to the plasma impedance in the ion source. Such a mini-ECR has been shown by the group at Peking University to produce high current ion beams having better stability and reliability for long term operation than conventional ECR sources. ${ }^{4}$ The PKU group has reported that this ECR source produces a normalized rms emittance of $\sim 0.13 \mu(=0.13 \mathrm{~mm}-\mathrm{mrad})^{5}$ for a $100 \mathrm{~mA} \mathrm{H}^{+}$ion beam at $50 \mathrm{kV}$. A total deuteron beam current of $83 \mathrm{~mA}$ has also been extracted at $50 \mathrm{kV}$ with a normalized rms emittance of less than $0.18 \mu$. This would imply that a total deuteron current up to $29 \mathrm{~mA}$ can be extracted at $25 \mathrm{kV}$ with a normalized rms emittance of less than $0.1 \mu$.

Another version of the mini-ECR is under development by Scientific Solutions (US DOE, Office of Nuclear Physics, SBIR grant DE-SC0004512). An advantage reported for this mini-ECR source is that it was designed to not have the "weekend effect" that plagues other ECR sources - it creates its own source of electrons and therefore always reliably starts working. ${ }^{6}$ 


\section{RFQ Accelerator}

The RFQ linac is a unique accelerator that can efficiently accelerate and focus a high current, low energy ion beam. Typically, it will accelerate more than $80 \%$ of a pulsed high current proton or deuteron beam injected into it at $25-35 \mathrm{keV}$ up to energies as high as $4 \mathrm{MeV}$ in less than $3 \mathrm{~m}$ length. While it can simultaneously bunch, focus, and accelerate an intense ion beam, all of its adjustable input parameters are somewhat insensitive to small fluctuations. The ion beam output current and energy are almost independent of the rf power level after a threshold value has been achieved. The input beam energy has a large acceptance, with little reduction in performance, and the resonant frequency of this robust, symmetric structure is easy to maintain by control of the cavity temperature. The RFQ generates bunched high-current ion beams and produces high-quality beam outputs with respect to emittance and energy spread, offering advantages over other technology as an accelerator for the production of neutrons.

The compact RFQ presented in this paper is designed to replace $\mathrm{D}(\mathrm{d}, \mathrm{n})$ sealed neutron generator tubes, which have a limited operational lifetime, particularly at the maximum neutron output. It operates at a high enough energy to also bombard a beryllium or lithium target. The initial cost of this unit is greater than a sealed tube, but the life-cycle costs are much less since the target lifetime is several years. It is totally field-serviceable, which offers a distinct advantage over sealed tube generators. This compact system can be mounted either horizontally or vertically, and a $20 \mathrm{~cm}$ beam tube can be used at the end of the linac for positioning the target in a moderator or shield assembly.

The operating parameters for the RFQ linac design are listed in Table 1. It is only $0.5 \mathrm{~m}$ long and accelerates deuterons from $25 \mathrm{keV}$ to $500 \mathrm{keV}$. The current limit is more than twice the operating output current, giving ample margin for the structure's performance.

Table 1. Mini-neutron RFQ design parameters.

\begin{tabular}{lll}
\hline Parameter & \multicolumn{2}{c}{ Value } \\
\hline Accelerated particle & $\mathrm{d}^{+}$ & $\mathrm{keV}$ \\
Input beam energy & 25 & $\mathrm{MeV}$ \\
Output beam energy (nominal) & 0.5 & $\mathrm{~cm}$ \\
Vane aperture & 0.159 & $\mathrm{kV}$ \\
Intervane voltage & 49.3 & $\mathrm{~kW}$ \\
RF input power & 30 & \\
Maximum vane modulation & 2.2 & $\mathrm{~mA}$ \\
Beam current limit & 28 & $\mu$ \\
Normalized beam acceptance & 0.47 & $\%$ \\
Beam transmission & 87 & $\mu \mathrm{s}$ \\
Beam pulse width & $10-100$ & $\mathrm{~Hz}$ \\
Pulse repetition rate & $100-1000$ & $\mu \mathrm{A}$ \\
Maximum target current & 1000 & $\mathrm{~m}$ \\
Accelerator length & 0.50 & $\mathrm{~kg}$ \\
Accelerator weight & $\sim 100$ & $\mathrm{kVA}$ \\
Electrical power requirement & 5.0 &
\end{tabular}


The standard RFQ beam dynamics design developed at Los Alamos National Laboratory was used for this system. The vane voltage is constant along its length, and the vane tip radius is constant. However, to reduce the vane length, the vane modulation (m) and synchronous phase (phi) are ramped even in the acceleration section. A small bore radius was used to reduce the rf power required to drive the RFQ while maintaining a current limit and acceptance suitable for this application.

Figure 2 shows the calculated phase space projections in both transverse planes at the output of the RFQ using 5000 macro particles. As seen in the figure, the beam exiting the RFQ is almost circular with a waist $<2 \mathrm{~mm}$ in diameter. The beam has a very small divergence at this point, allowing the beam to drift through a small diameter beam tube of $20 \mathrm{~cm}$ length to a target that can easily be cooled and placed in a small moderator or collimator assembly.
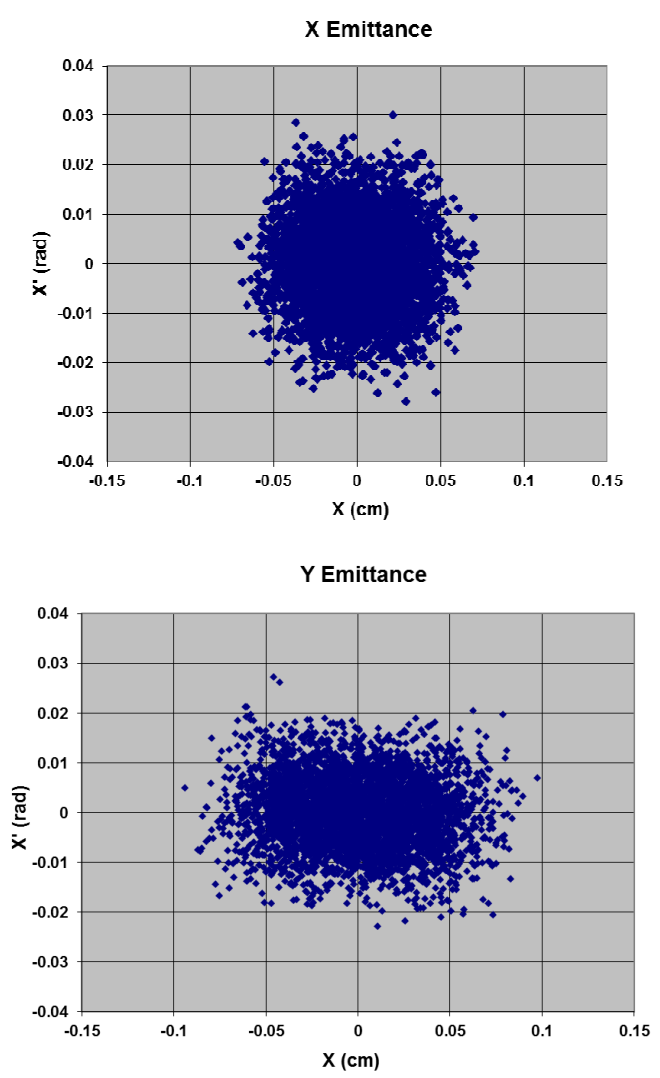

Fig. 2. RFQ output beam emittance in both transverse planes.

Figure 3 plots the beam transmission of the RFQ calculated as a function of the intervane voltage (i.e., input rf power), clearly showing that the transmission is almost constant above the design vane voltage. The RFQ output energy is fixed after reaching 
this critical rf power level and does not change as the power is raised above that value. Hence the RFQ output beam will have a high level of current and energy stability with respect to small variations in the input rf power.

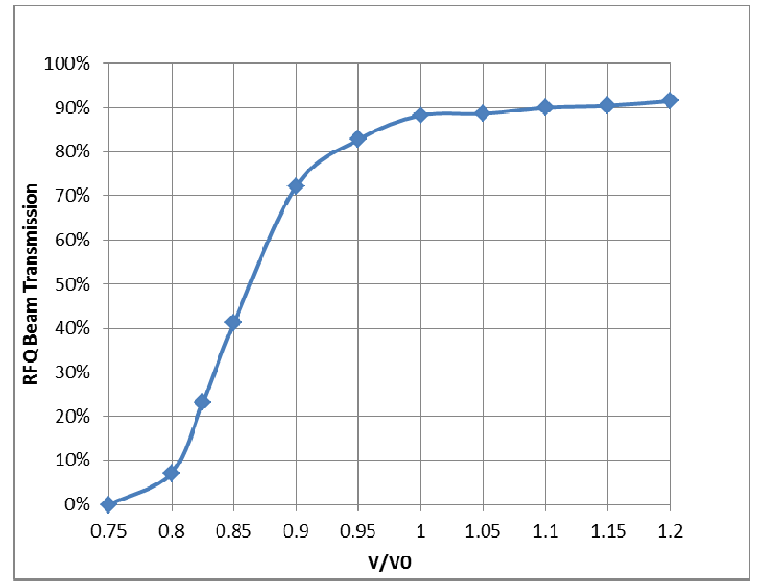

Fig. 3. RFQ beam transmission as a function of intervane voltage.

\section{Injector Electrode Matching Design with IGUN}

We have designed a combined extraction/matching system for a compact high current deuterium ECR ion source injecting a beam into this high frequency RFQ structure, as shown in Fig. 4. The design allows a $\mathrm{d}^{+}$beam of $\sim 12 \mathrm{~mA}$ to be injected at a modest ion source voltage of $25 \mathrm{kV}$. The end wall of the RFQ resonator serves as the ground electrode for the ion source, resembling direct plasma injection (DPI). ${ }^{7}$

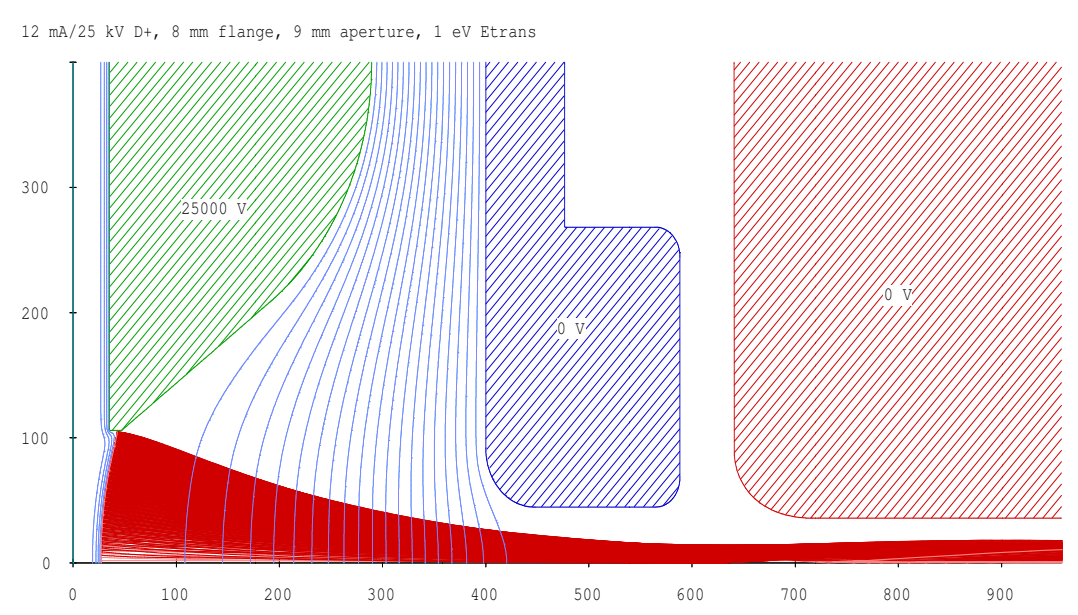

Fig. 4. Beam extraction and matching electrodes for direct injection into the RFQ. 
For this design, we have used the unique features of IGUN $^{8}$ to take into account the electrostatic field between the ion source and the RFQ end wall, the stray magnetic field of the ECR source, the defocusing space charge of the low energy deuteron beam, and the rf focusing in the fringe field between the RFQ vanes and the RFQ flange. ${ }^{9}$ The result is an ion beam matching system of minimum size. The only disadvantages are (1) the beam current is fixed, and (2) there are no tuning elements, such as focusing lenses, for optimizing the current into the RFQ.

In designing the matching electrodes, the calculations were restricted to the region between the plasma meniscus and the RFQ matching point as shown in Fig. 5. This matching plane is where the acceptance (rms-emittance and ellipse Twiss parameters) has been defined by the RFQ design, which was presented above in Section 3. In this region, the convergence of the ion beam is directly controlled by the curvature of the meniscus, which in turn is controlled by the accelerating field penetrating to the meniscus. We have used the diameter of the meniscus, the angle of the plasma electrode at the meniscus edge, the curvature of the plasma electrode towards the RFQ entrance flange, and the distance of this flange from the meniscus in order to provide an optimized solution for 12 $\mathrm{mA} \mathrm{d}^{+}$ions being accelerated by a $25 \mathrm{kV}$ gap voltage and matched properly into the acceptance of the RFQ, as seen in Fig. 6.

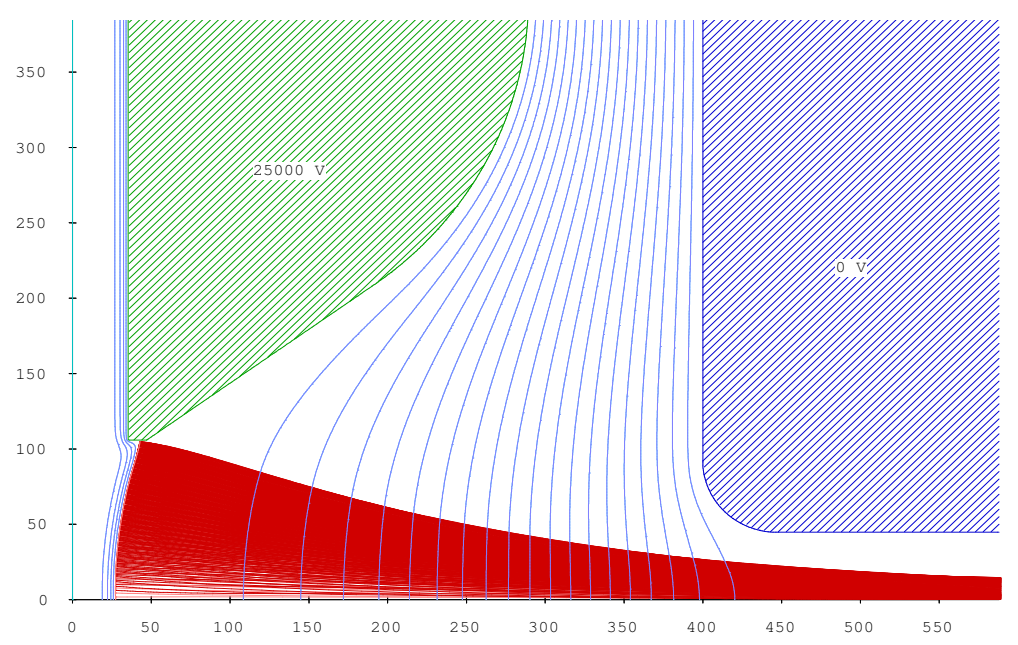

Fig. 5. Beam extraction from the plasma meniscus to the RFQ matching point.

IGUN provides assistance for this optimization in two different ways. In the initial design calculations, if not all of the current from the ion source is focused inside the acceptance, IGUN reports the percentage of current that is accepted. Each modification of the electrode design then results in an improvement (accepted) or in a reduction of this value (rejected). After all current has been successfully matched into the acceptance ellipse (shown in Fig. 6 as the ellipse with the largest area), IGUN calculates the smallest ellipse that contains all of the trajectories at the matching point and has the same Twiss 
parameters as the acceptance ellipse. This ellipse is shown in Fig. 6 as the one with the same tilt and eccentricity as the acceptance but lying inside of it. The area of this ellipse compared to the area of the acceptance ellipse is then a direct measure of the improvements in any further optimization, reaching $69 \%$ in this case. The inner ellipses show the rms and $4 x$ rms phase space of the beam itself.

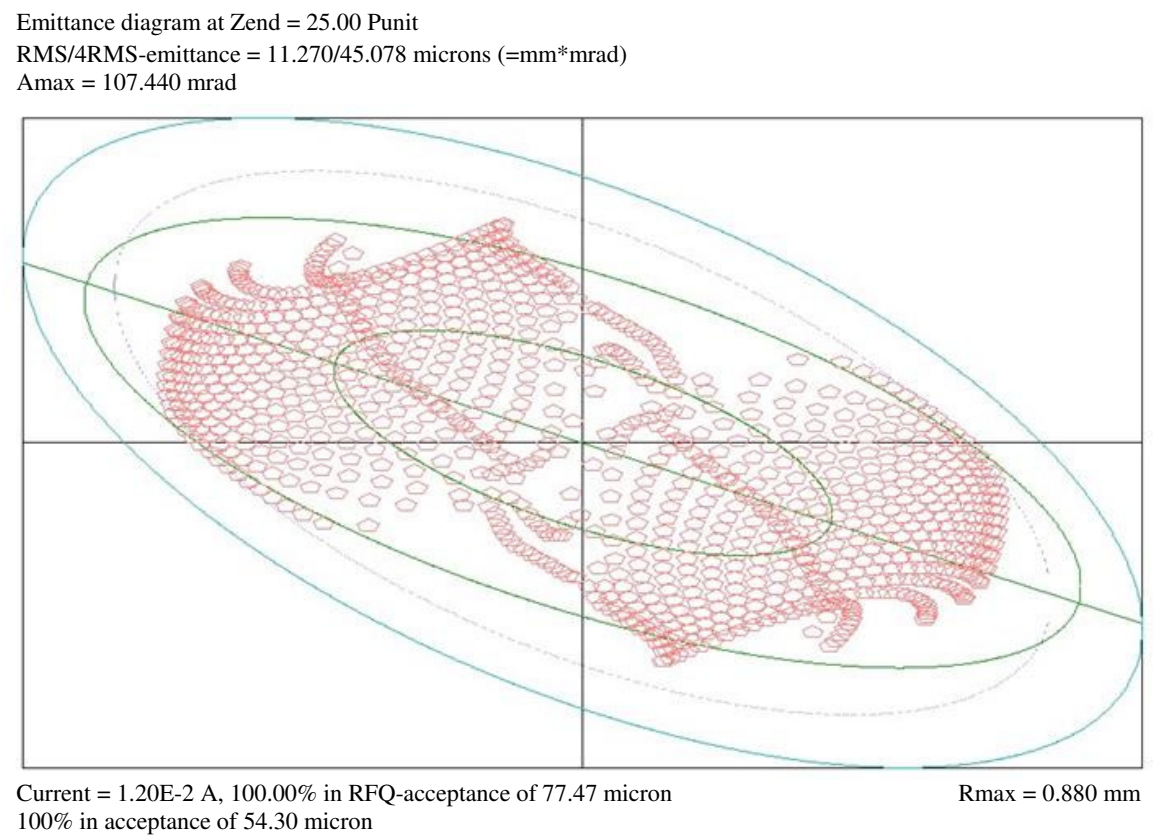

Fig. 6. Phase space for optimized beam from the ion source matched into the RFQ. (See text above for explanation of ellipses shown in this figure.)

\section{Conclusion}

RFQ-based neutron generators world-wide have demonstrated to be well-suited for a number of applications and offer unique capabilities not available from other neutron sources. The compact RFQ-based system presented in this paper will now make them competitive with the conventional sealed tube neutron generators used in many industrial applications. This compact unit is only now possible through the use of a new mini-ECR ion source and direct injection optics into a small RFQ linac driven by a compact solidstate rf system.

\section{References}

1. M. R. Hawkesworth, Atomic Energy Review 15 (2), 169 (1977).

2. N. Colonna et al., AIP Conf. Proc. 475, 1045 (1998).

3. R. Hamm, Proc. SPIE 4142, 39 (2000), doi:10.1117/12.410581.

4. S. X. Peng et al., Proc. ECRIS 2010, Grenoble, France (2013), p. 104, JaCow website (http://accelconf.web.cern.ch/accelconf/). 
5. R. Becker and W. B. Herrmannsfeldt, Rev. Sci. Instr. 77, 03 B907 (2006).

6. W. Cornelius, private communication.

7. M. Okamura et al., Rev. Sci. Instr. 73 (2), 761 (2002).

8. R. Becker and W. B. Herrmannsfeldt, Rev. Sci. Instr. 63 (4), 2756 (1992).

9. R. Becker and R. A. Jameson, Nucl. Instr. Meth. A 558, 205 (2006). 